\title{
Conjugate Fabry-Perot Cavity Pair for Improved Astro-Comb Accuracy
}

\section{Citation}

Li, Chih-Hao, Guoqing Chang, Alexander G. Glenday, Nicholas Langellier, Alexander Zibrov, David F. Phillips, Franz X. Kärtner, et al. 2012. Conjugate Fabry-Perot Cavity Pair for Improved AstroComb Accuracy. Optics Letters 37, no. 15: 3090-3092.

\section{Published Version}

doi:10.1364/OL.37.003090

\section{Permanent link}

http://nrs.harvard.edu/urn-3:HUL.InstRepos:11878773

\section{Terms of Use}

This article was downloaded from Harvard University's DASH repository, and is made available under the terms and conditions applicable to Other Posted Material, as set forth at http:// nrs.harvard.edu/urn-3:HUL.InstRepos:dash.current.terms-of-use\#LAA

\section{Share Your Story}

The Harvard community has made this article openly available.

Please share how this access benefits you. Submit a story.

\section{Accessibility}




\title{
Conjugate Fabry-Perot cavity pair for improved astro-comb accuracy
}

\author{
Chih-Hao Li, ${ }^{1,+* *}$ Guoqing Chang, ${ }^{2,+}$ Alexander G. Glenday, ${ }^{1}$ Nicholas Langellier, ${ }^{3}$ \\ Alexander Zibrov, ${ }^{1}$ David F. Phillips, ${ }^{1}$ Franz X. Kärtner, ${ }^{2,4}$ \\ Andrew Szentgyorgyi, ${ }^{1}$ and Ronald L. Walsworth ${ }^{1,3}$ \\ ${ }^{1}$ Harvard-Smithsonian Center for Astrophysics, Harvard University, 60 Garden St., Cambridge, Massachusetts 02138, USA \\ ${ }^{2}$ Department of Electrical Engineering and Computer Science and Research Laboratory of Electronics, \\ Massachusetts Institute of Technology, Cambridge, Massachusetts 02139, USA \\ ${ }^{3}$ Department of Physics, Harvard University, Cambridge, Massachusetts 02138, USA \\ ${ }^{4}$ Center for Free-Electron Laser Science, DESY and Department of Physics, University of Hamburg, \\ Notkestraße 85, D-22607 Hamburg, Germany \\ *Corresponding author: chli@cfa.harvard.edu
}

Received May 10, 2012; revised June 15, 2012; accepted June 16, 2012; posted June 18, 2012 (Doc. ID 168342); published July 19, 2012

\begin{abstract}
We propose a new astro-comb mode-filtering scheme composed of two Fabry-Perot cavities (coined "conjugate Fabry-Perot cavity pair"). Simulations indicate that this new filtering scheme makes the accuracy of astrocomb spectral lines more robust against systematic errors induced by nonlinear processes associated with power-amplifying and spectral-broadening optical fibers. (c) 2012 Optical Society of America
\end{abstract}

OCIS codes: $120.2230,120.5060$.

Astro-combs are broadband $(\sim 100 \mathrm{~nm})$, wide-linespacing ( $>10 \mathrm{GHz})$ optical frequency combs referenced to atomic clocks and optimized for wavelength calibration of astrophysical spectrographs. Astro-combs are expected to increase the accuracy and long-term stability of astrophysical spectroscopy [1-9], with applications including the detection and characterization of extra-solar planets (exoplanets) and direct measurement of the acceleration of the universe. In particular, searches for Earth-like exoplanets around Sun-like stars using precision stellar radial velocity (RV) measurements require $<5 \mathrm{~cm} / \mathrm{s} \mathrm{RV}$ accuracy and long-term stability, which is equivalent to $100 \mathrm{kHz}(50 \mathrm{kHz})$ accuracy of Doppler shift measurements of stellar light at $500 \mathrm{~nm}(1 \mu \mathrm{m})$ over several-year observation periods.

An astro-comb consists of an octave-spanning laser frequency comb ("source comb"), typically operating in the near-infrared with spectral line spacing $<1 \mathrm{GHz}$ [10], integrated with a coherent wavelength-shifting mechanism (such as a doubling crystal or photonic crystal fiber), a mode-filtering Fabry-Perot cavity (FPC), which effectively increases the line spacing to $>10 \mathrm{GHz}$, and power-amplifying and spectral-broadening optical fibers. Several prototype astro-combs have recently been developed and tested with astrophysical spectrographs [5-9,11]. These tests have shown that the mode-filtering systems must be improved to provide adequate suppression of unwanted spectral lines ("side modes") in the presence of nonlinear processes in optical fibers. Here, we propose a new mode-filtering design, using two FPCs of incommensurate length, that improves the performance of astro-combs containing nonlinear elements such as photonic crystal fiber and fiber amplifiers by minimizing the optical phase shifts associated with the FPCs.

The source comb spectrum is composed of many narrow lines equally spaced in frequency, with a line spacing given by the laser's pulse repetition frequency, $f_{r}$, (typically $<1 \mathrm{GHz}$ ). A FPC mode-filter, with free spectral range (FSR) equal to $M f_{r}$, where $M \approx 10-100$, passes every $M$ th source comb line and suppresses intermediate lines such that the astro-comb spectrum has line spacing $M$ times larger than the source comb. This wide line spacing is required for high-resolution astrophysical spectrographs with resolving power $R=\lambda / \Delta \lambda \approx 50,000$ to 100,000 , corresponding to a resolution of 5 to $10 \mathrm{GHz}$ in the optical spectral range, to resolve the lines.

Because the spectrograph fails to resolve individual source comb lines, finite suppression of unwanted side modes by the FPC shifts the centroid of astro-comb lines as measured on the spectrograph. The shift, expressed as a radial velocity, $\delta \mathrm{RV}$, may be estimated by

$$
\frac{\delta R V}{c}=\frac{\delta f_{A}}{f_{A}} \approx \frac{f_{r}}{f_{A}} \sum_{k=-M / 2}^{M / 2} k \cdot I_{k} / \sum_{k=-M / 2}^{M / 2} I_{k},
$$

where $f_{A}$ is the astro-comb line frequency, $\delta f_{A}$ is the shift of the astro-comb line as measured on the spectrograph, $I_{k}$ is the intensity of the $k$ th side mode (the source comb line $k$ repetition frequencies away from the astro-comb line), and $c$ is the speed of light [11,12]. To calibrate the spectrograph with an accuracy of $100 \mathrm{kHz}$ with an astrocomb, the intensity of the side modes must be suppressed or controlled to $<10^{-4}$ of the astro-comb peak intensity $[1,11]$. This suppression is nominally achievable with FPCs of moderate finesse, but can be significantly degraded in the presence of nonlinear optical elements, as discussed below.

An FPC suppresses the intensity of transmitted light of wavelength $\lambda$ by:

$$
I_{\text {out }} / I_{\text {in }}=(1-R)^{2} /\left[(1-R)^{2}+4 R \sin ^{2}(\delta(\lambda) / 2)\right]
$$

where $R$ is the FPC mirror reflectivity and $\delta(\lambda)=$ $2\left(2 \pi l / \lambda+\phi_{m}\right)$ is the cavity roundtrip phase delay, with $l$ the cavity length and $\phi_{m}$ the wavelength-dependent mirror penetration depth [Fig. 1(a)]. (Note that there is an additional phase, $\phi_{g}$, the Gouy phase, associated with 
each transverse mode of the cavity, which is a wavelength independent constant; for curved-mirror cavities this phase leads to nondegenerate transverse modes and thus requires careful mode matching.) However, when fiber amplifiers and nonlinear broadening fibers are used after an FPC to achieve broadband (>100 nm) coverage [ $\underline{6}-\underline{8}$ ], this highly nonlinear process typically leads to a degradation of the side mode suppression, mostly due to four-wave-mixing (FWM) that redistributes power among comb lines $[6,12]$. Problematically, the FPC imposes a phase shift (the cavity phase),

$$
\psi=\tan ^{-1}(R \sin \delta /(1-R \cos \delta))
$$

as shown in Fig. 1(b), on the source comb lines. Because FWM processes are phase sensitive [12], the cavity phase leads to a difference in the degradation of side mode suppression between upper and lower side modes of an astro-comb line, and thus to a systematic shift of the centroid of the astro-comb line as measured on an astrophysical spectrograph. As a result, two FPCs with much higher finesse [7], or three or more FPCs [8] are often necessary to achieve the required accuracy when fiber power amplifiers and spectral broadening fibers are used after an FPC.

To reduce systematic calibration errors in astro-combs induced by FWM, we propose a new filtering scheme using two series FPCs of differing FSR, coined a "conjugate Fabry-Perot cavity (cFPC) pair," which minimizes the FPC cavity phase shift on the side modes and therefore minimizes imbalance in the side-mode suppression and the resultant shift in the measured line centroid. The two FPCs are composed of mirror pairs of equal reflectivity and dispersion but with different FSRs given by $\Delta_{1}=M \cdot f_{r}$ and $\Delta_{2}=M /(M-1) \cdot f_{r}$. The cavities are operated in vacuum to eliminate the effects of air dispersion, which is critical to eliminate additional dispersion in the longer cavity. Astro-comb lines are on resonance with both FPCs, so the roundtrip phase $\delta_{A} \approx 0(\bmod 2 \pi)$.

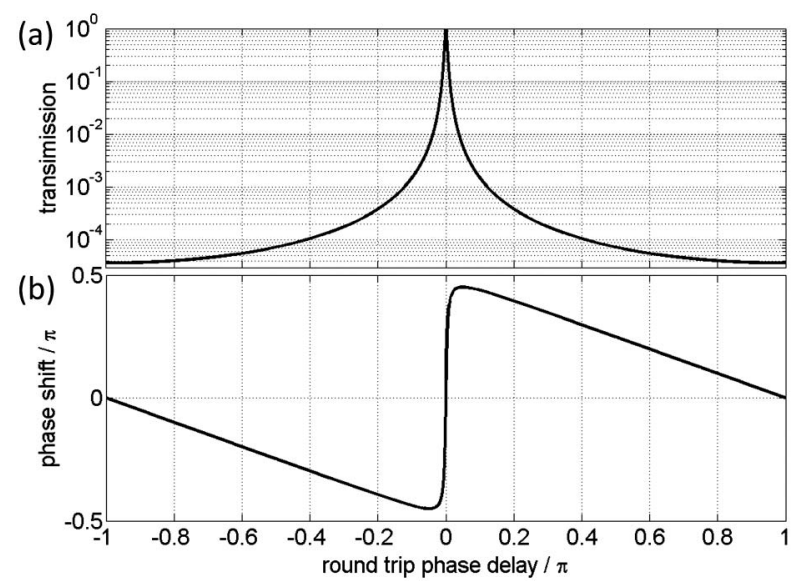

Fig. 1. (a) Transmission and (b) phase shift imposed on incident light of wavelength $\lambda$ by a Fabry-Perot cavity (FPC), as a function of cavity roundtrip phase delay, $\delta(\bmod 2 \pi)$. Plots are for a FPC composed of two identical mirrors of reflectivity $R=98.8 \%$. Critical to conjugate FPC (cFPC) performance is that FPC transmission (phase shift) be an even (odd) function of $\delta$.
For the $p$ th side mode with frequency $p \cdot f_{r}$ higher than the nearest astro-comb line, (where $p$ is a positive or negative integer), $\delta_{p(1,2)} \approx 2 \pi \cdot p \cdot f_{r} / \Delta_{(1,2)}(\bmod 2 \pi)$, where $\Delta_{(1,2)}$ is the FSR of the first or second FPC. The roundtrip phase delays from the cavities of a cFPC pair are $\delta_{p, 1} \approx$ $2 \pi \cdot p / M$ and $\delta_{p, 2} \approx-2 \pi \cdot p / M=-\delta_{p, 1}$. For an FPC, the transmission is an even function of $\delta$ [as shown in Eq. (2) and Fig. 1(a)] and the total cavity phase is an odd function of $\delta$ [as shown in Eq. (3) and Fig. 1(b)]. Therefore, the total cavity phase of the $p$ th side mode resulting from the cFPC pair is $\psi_{1}+\psi_{2} \approx 0$ (Fig. 2). Meanwhile, the suppression of side modes is not compromised, as suppression at the frequencies of the side modes is the same as for two identical FPCs with $\Delta=M \cdot f_{r}$. As a result, the systematic error in astro-comb lines following spectral broadening of an astro-comb spectrum filtered by a cFPC pair is minimized because the cavity phase from the filtering cavity is near zero [12].

We performed numerical simulations to demonstrate the improvement on the accuracy of an astro-comb with cFPC pairs, compared to an astro-comb with two identical FPCs $\left(\Delta=M \cdot f_{r}\right)$. The source comb is modeled as a $\mathrm{Yb}$ fiber laser frequency comb with a pulse duration of $60 \mathrm{fs}$, corresponding to a bandwidth of $50 \mathrm{~nm}$, pulse repetition rate of $250 \mathrm{MHz}$, pulse energy of $60 \mathrm{pJ}$ and center wavelength of $1.06 \mu \mathrm{m}$. The astro-comb line spacing is taken to be $16 \mathrm{GHz}(M=64)$, the FPC mirror reflectivity is $98.8 \%$ (cavity finesse $=260$ ), and we have assumed no additional losses associated with the cavities leading to a pulse energy at the output of the cFPC pair of $0.015 \mathrm{pJ}$. The spectral broadening fiber is a $20 \mathrm{~mm}$ SF6 photonic crystal fiber (PCF) with mode field diameter of $1.7 \mu \mathrm{m}$ and nonlinear parameter of $570 \mathrm{~W}^{-1} \mathrm{~km}^{-1}[\underline{13}]$. The nonlinear spectral broadening of ultrashort pulses in an optical fiber is modeled by the generalized nonlinear Schrödinger equation [14]. We performed simulations for both identical FPCs

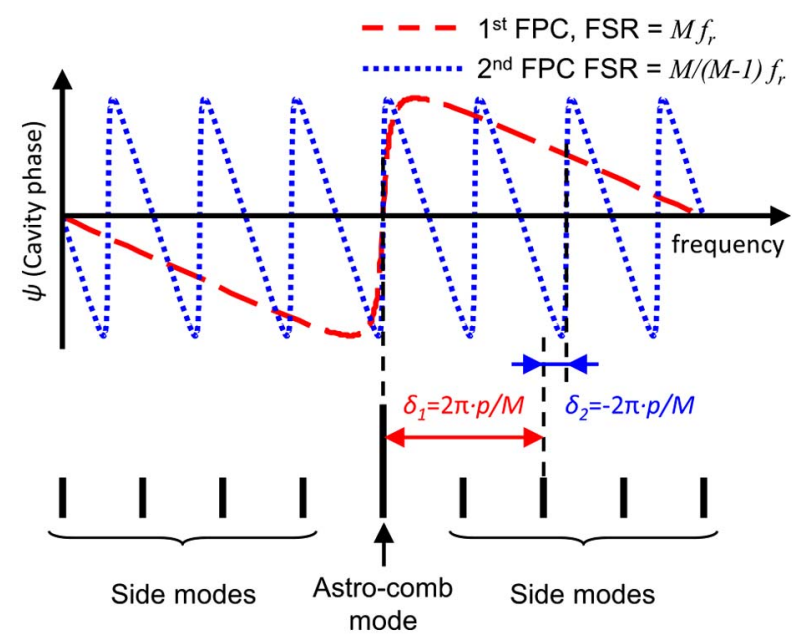

Fig. 2. (Color online) Roundtrip phase delay imposed by conjugate Fabry-Perot cavity (cFPC) pair on (desired) astro-comb mode and (unwanted) side modes. Dashed red and dotted blue curves are cavity phases imposed by first and second FPCs, respectively. For all side modes, roundtrip phase delays and cavity phases of the two FPCs are equal in magnitude but opposite in sign. As a result, overall cavity phase imposed by the cFPC pair is zero for all side modes. (Phase shifts are shown for $M=8$.) 

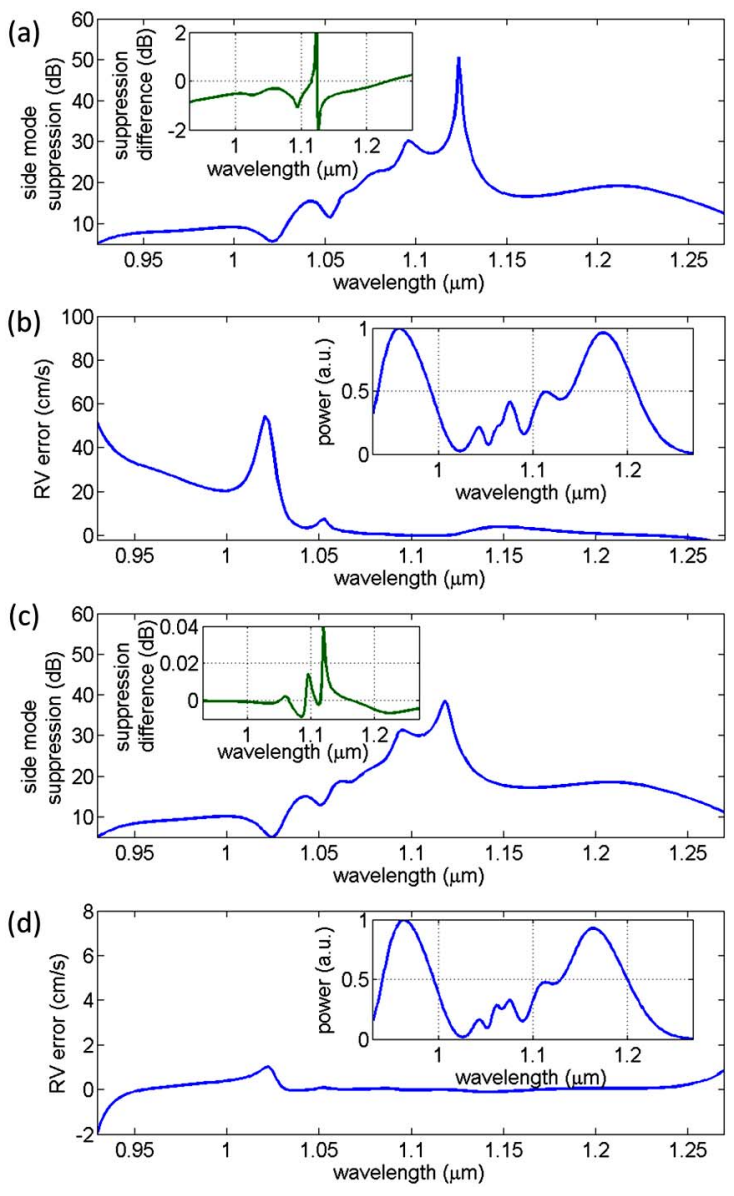

Fig. 3. (Color online) Simulation of astro-comb accuracy for two different filtering schemes: (a), (b) two identical FPCs, and (c), (d) conjugate FPC (cFPC) pair. cFPC pair provides 30-fold improvement in side mode filtering after spectral broadening, compared to two identical FPCs, because cFPC suppression degradation on blue and red side modes is nearly identical.

[Figs. 3(a), 3(b)] and cFPC pairs Figs. 3(c), 3(d). Details of the simulation method are presented in [12]. We find that side mode suppression with cFPC pairs is more balanced, and thus systematic shifts of desired astro-comb lines are reduced as measured on an astrophysical spectrograph, compared to two identical FPCs, though some degradation of side mode suppression is still present with the cFPC pairs. The systematic shift of astro-comb line centers with a cFPC pair is typically $<1 \mathrm{~cm} / \mathrm{s}$, expressed as RV error [from Eq. (1)], which is a 30-fold improvement over an astro-comb based on two identical FPCs. The simulations presented in this work are performed at $1 \mu \mathrm{m}$. Similar results are expected when wavelength shifting, as well as spectral broadening, is used to produce a visible wavelength astro-comb.

In conclusion, we propose a new mode-filtering scheme for astro-combs based on a conjugate FabryPerot cavity (cFPC) pair composed of two FPCs with different FSRs: $\mathrm{c} \Delta_{1}=M f_{r}$ and $\Delta_{2}=M /(M-1) f_{r}$, which achieves an astro-comb mode spacing of $M f_{r}$. The cFPC pair imposes a minimal overall cavity phase on the side modes of an astro-comb and therefore minimizes the imbalanced degradation of side mode suppression resulting from nonlinear processes associated with power amplifying and spectral broadening optical fibers. Simulations show that the accuracy of astro-comb lines with a cFPC pair has 30 times smaller systematic errors induced by nonlinear processes than an astro-comb with two identical FPCs.

This work was performed with support from National Science Foundation (NSF) grants AST-0804441, ATI0905214, and ATI-1006503; NASA grants NNX09AC92G and NNX10AE68G; and internal support from the Smithsonian Astrophysical Observatory.

These authors contributed equally to the work.

\section{References}

1. M. T. Murphy, T. Udem, R. Holzwarth, A. Sizmann, L. Pasquini, C. Araujo-Hauck, H. Dekker, S. D’Odorico, M. Fischer, T. W. Hänsch, and A. Manescau, Mon. Not. R. Astron. Soc. 380, 839 (2007).

2. C.-H. Li, A. J. Benedick, P. Fendel, A. G. Glenday, F. X. Kärtner, D. F. Phillips, D. Sasselov, A. Szentgyorgyi, and R. L. Walsworth, Nature 452, 610 (2008).

3. T. Steinmetz, T. Wilken, C. Araujo-Hauck, R. Holzwarth, T. W. Hänsch, L. Pasquini, A. Manescau, S. D'Odorico, M. T. Murphy, T. Kentischer, W. Schmidt, and T. Udem, Science 321, 1335 (2008).

4. D. A. Braje, M. S. Kirchner, S. Osterman, T. Fortier, and S. A. Diddam, Eur. Phys. J. D 48, 57 (2008).

5. A. J. Benedick, G. Chang, J. R. Birge, L.-J. Chen, A. G. Glenday, C.-H. Li, D. F. Phillips, A. Szentgyorgyi, S. Korzennik, G. Furesz, R. L. Walsworth, and F. X. Kärtner, Opt. Express 18, 19175 (2010).

6. F. Quinlan, G. Ycas, S. Osterman, and S. A. Diddams, Rev. Sci. Instrum. 81, 063105 (2010).

7. G. G. Ycas, F. Quinlan, S. A. Diddams, S. Osterman, S. Mahadevan, S. Redman, R. Terrien, L. Ramsey, C. F. Bender, B. Botzer, and S. Sigurdsson, Opt. Express 20, 6631 (2012).

8. T. Wilken, G. L. Curto, R. A. Probst, T. Steinmetz, A. Manescau, L. Pasquini, J. I. G. Hernandez, R. Rebolo, T. W. Hänsch, T. Udem, and R. Holzwarth, Nature 485, 611 (2012).

9. D. F. Phillips, A. G. Glenday, C.-H. Li, C. Cramer, G. Furesz, G. Chang, A. J. Benedick, L.-J. Chen, F. X. Kärtner, S. Korzennik, D. Sasselov, A. Szentgyorgyi, and R. L. Walsworth, Opt. Express 20, 13711 (2012).

10. T. Udem, R. Holzwarth, and T. W. Hänsch, Nature 416, 233 (2002).

11. C.-H. Li, A. G. Glenday, A. J. Benedick, G. Chang, L.-J. Chen, C. Cramer, P. Fendel, G. Furesz, F. X. Kärtner, S. Korzennik, D. F. Phillips, D. Sasselov, A. Szentgyorgyi, and R. L. Walsworth, Opt. Express 18, 13239 (2010).

12. G. Chang, C.-H. Li, D. F. Phillips, R. L. Walsworth, and F. X. Kärtner, Opt. Express 18, 12736 (2010).

13. H. Hundertmark, S. Rammler, T. Wilken, R. Holzwarth, T. W. Hänsch, and P. St. J. Russell, Opt. Express 17, 1919 (2009).

14. G. P. Agrawal, Nonlinear Fiber Optics, 3rd ed. (Academic, 2001). 